\title{
Metallic Stripes in High-Temperature Superconductors
}

\author{
M. I. Salkola ${ }^{1 a}$, V. J. Emery ${ }^{2}$, and S. A. Kivelson ${ }^{3 b}$ \\ ${ }^{1}$ Theoretical Division, Los Alamos National Laboratory, Los Alamos, New Mexico 87545 \\ ${ }^{2}$ Department of Physics, Brookhaven National Laboratory, Upton, New York 11973-5000 \\ ${ }^{3}$ Institute for Theoretical Physics, University of California, Santa Barbara, California 93106 \\ PECENED
}

(November 23, 1995)

A phenomenological approach is applied to explore signatures of disordered charge stripes and antiphase spin domains in single-particle properties of the high-temperature superconductors. Stripe phases are shown to explain many experimentally observed unusual features measured in angleresolved photoemission and optical spectroscopy. It is argued that disordered and fluctuating stripe phases are a common feature of high-temperature superconductors, supported by the additional evidence from neutron scattering and NMR.

\section{INTRODUCTION}

One of the emerging new paradigms of hightemperature superconductors is that their normal-state properties are inherently connected to disordered or fluctuating stripe phases [1]. In this paper, we show that single-particle properties of a disordered stripe phase can account for exotic features of the spectral density measured by angle-resolved photoemission spectroscopy (ARPES) in $\mathrm{Bi}_{2} \mathrm{Sr}_{2} \mathrm{CaCu}_{2} \mathrm{O}_{8+x}$ (for which the best data is available). In particular, we compute the spectral density in a background of disordered stripes and show that it reproduces the experimentally-observed shape of the Fermi surface, the existence of nearly dispersionless states at the Fermi energy ("flat bands"), and the appearance of weak additional states ("shadow bands") [2-5], features which have no natural explanation within conventional band theory. Finally, we comment briefly on the implications of partially ordered spin domains for NMR experiments.

Recent elastic neutron-scattering experiments [6] in $\mathrm{La}_{1,6-x} \mathrm{Nd}_{0.4} \mathrm{Sr}_{x} \mathrm{CuO}_{4}$ show that the suppression of superconductivity in the neighborhood of $x=\frac{1}{8}$ is associated with the formation of an ordered array of charged stripes which form antiphase domain walls between antiferromagnetically ordered spins in the $\mathrm{CuO}_{2}$ planes. This observation provides the long-sought explanation of the behavior [7] of the $\mathrm{La}_{2} \mathrm{CuO}_{4-\delta}$ family of compounds near to $\frac{1}{8}$ doping, and strongly supports the idea of stripe phases.

Antiphase spin domains in $\mathrm{La}_{1.6-x} \mathrm{Nd}_{0.4} \mathrm{Sr}_{x} \mathrm{CuO}_{4}$ are indicated by a set of resolution-limited peaks in the magnetic structure factor at wave vectors $\left(\frac{1}{2} \pm \epsilon, \frac{1}{2}\right)$ and $\left(\frac{1}{2}, \frac{1}{2} \pm \epsilon\right)[6]$. The associated charge stripes give rise to peaks in the nuclear structure factor at wave vectors $( \pm 2 \epsilon, 0)$ and $(0, \pm 2 \epsilon)$ [8]. As mentioned by Tranquada et al. [6], it is natural to interpret the inelastic peaks in the magnetic structure factor which occur [9] at similar locations in reciprocal space in superconducting samples of $\mathrm{La}_{2-x} \mathrm{Sr}_{x} \mathrm{CuO}_{4-\delta}$ as evidence of "extended domains"
[10] of stripe fluctuations, in which the stripes are oriented along vertical or horizontal $\mathrm{Cu}-\mathrm{O}$ bond directions respectively.

In the early days of high-temperature superconductivity, it was shown that holes added to an antiferromagnet form an insulating stripe phase via a Fermi-surface instability [11], which leads to a reduced density of states, or a gap, at the Fermi energy. An alternative mechanism for stripe phases is the competition between phase separation [12] (i.e., the tendency of an antiferromagnetic insulator to expel doped holes) and the long-range part of the Coulomb interaction. In this case, the stripes may be either ordered, quantum melted, or disordered by quenched randomness [13]. The charge forms an array of metallic stripes, with a period that is determined by the energetics of phase separation and is unrelated to nesting vectors of the Fermi surface. The charge structures, in turn, drive the modulation of the antiferromagnetic order. The experiments [6] on $\mathrm{La}_{1.6-x} \mathrm{Nd}_{0.4} \mathrm{Sr}_{x} \mathrm{CuO}_{4}$ clearly favor the latter point of view. The ordering wave vectors do not nest the Fermi surface, and the ordered system has partially-filled hole bands associated with the stripes. Moreover the magnetic peaks first develop below the charge-ordering temperature $[6,14]$. Our interpretation of the ARPES experiments on $\mathrm{Bi}_{2} \mathrm{Sr}_{2} \mathrm{CaCu}_{2} \mathrm{O}_{8+x}$ lends further support to this conclusion: we find an increased density of states corresponding to the flat bands seen in the experiments.

\section{STRIPE PHENOMENOLOGY}

Our objective is to determine a phenomenological band structure for electrons moving in an effective potential generated by charge stripes and antiphase spin domains. The phenomenological one-body Hamiltonian is given by:

$$
H=-t \sum_{\left(l^{\prime}\right)^{\prime} \sigma}\left(c_{l_{\sigma}}^{\dagger} c_{l^{\prime} \sigma}+H . c .\right)+\sum_{l \sigma} V_{\sigma}\left(\mathbf{R}_{l}\right) n_{l \sigma},
$$


where the first term is the nearest-neighbor hopping on a square lattice and the second one describes the interaction with the effective stripe potential. Here, $c_{l \sigma}$ annihilates an electron of spin $\sigma= \pm$ at site $\mathbf{R}_{l}$ and $n_{l \sigma}=c_{l \sigma}^{\dagger} c_{l \sigma}$. The effective potential is given by:

$$
V_{\sigma}(\mathbf{R})=\rho(\mathbf{R})+\sigma S(\mathbf{R}) e^{i \mathbf{Q} \cdot \mathbf{R}},
$$

where $\mathbf{Q}=\left(\frac{\pi}{a}, \frac{\pi}{a}\right)$ and $a$ is the lattice spacing. Specifically, for vertical stripes, we. use the forms $\rho(x, y)=$ $\rho_{0} \sum_{n} \operatorname{sech}\left[\left(x-x_{n}\right) / \xi_{c}\right]$ and $S(x, y)=S_{0} \prod_{n} \tanh [(x-$ $\left.\left.x_{n}\right) / \xi_{s}\right]$, where $\mathbf{R}=(x, y), x_{n}$ are fixed centers of the stripes, and the parameters $\rho_{0}, S_{0}, \xi_{c}$, and $\xi_{s}$ determine the amplitude of the charge and spin modulation and whether the stripes are narrow or broad.

\section{ARPES AND STRIPE PHASES}

According to the usual interpretation [15], the measured photo-current in a photoemission experiment is the product of the electronic spectral density $A_{-}(\mathbf{k}, \epsilon)$ for the removal of one electron from the system and a slowly varying matrix element which reflects the photon polarization selection rules. This spectral density can be written as $A_{-}(\mathbf{k}, \epsilon)=f(\epsilon) A(\mathbf{k}, \epsilon)$, where $f(\epsilon)=1 /\left[e^{\left(\epsilon-\epsilon_{F}\right) / k_{B} T}+1\right]$ is the Fermi function, $\epsilon_{F}$ is the Fermi energy, and $A(\mathbf{k}, \epsilon)=-(1 / \pi) \operatorname{Im} G\left(\mathbf{k}, \epsilon+i 0^{+}\right)$ is the spectral density of the one-electron Green's function $G(\mathbf{k}, t)=-i\left\langle T c_{\mathbf{k} \sigma}(t) c_{\mathbf{k} \sigma}^{\dagger}(0)\right\rangle$.

\section{A. An ordered stripe phase}

First, consider vertical stripes condensed into a regular array: $\rho(x+\ell)=\rho(x)$ and $s(x+2 \ell)=s(x)$, where $\ell$ is the separation between stripes. Results will be presented for bond-centered stripes, $x_{n}=n \ell+a / 2$ with $\ell / a$ integer, but they are largely insensitive to this assumption. For even $\ell / a$, the unit cell size is $(2 \ell / a) \times 2$ so the bandstructure is computed by diagonalizing a $(4 \ell / a) \times(4 \ell / a)$ matrix for each $\mathrm{k}$-vector.

Figure 1 shows the spectral density $A_{-}$(integrated over an energy window $\Delta \epsilon=t / 30$ about $\epsilon_{F}$ ) as a function of $\mathbf{k}$. For illustration, we have used the parameters $\rho_{0}=-t / 2, S_{0}=2 t, \xi_{c}=a$, and $\xi_{s}=2 a$, for which the Hamiltonian in Eq. (1) solves Hartree-Fock theory for the Hubbard model with $U / t=4-5$. However, to describe $\mathrm{La}_{1.6-x} \mathrm{Nd}_{0.4} \mathrm{Sr}_{x} \mathrm{CuO}_{4}$ [6] at $x=\frac{1}{8}$ doping, we choose $\ell / a=4$, which does not minimize the HartreeFock energy.

The fine features of the Fermi surface reflect the energy gaps at points spanned by the wave vectors $\left(\frac{1}{2} \pm \epsilon, \frac{1}{2}\right)$ of the spin order and $( \pm 2 \epsilon, 0)$ of the charge order, where $\epsilon=a / 2 \ell$ : they are generated by the multiple foldings of the energy band in the first Brillouin zone by the effective stripe potential $V_{\sigma}(\mathbf{R})$. Figure 1 also shows shadow bands - weak features of the Fermi surface created by the local doubling of the unit cell in the regions between the stripes.

In Fig. 2, the spectral density $A_{-}$is shown for $\ell / a=10$ at $x=\frac{1}{20}$ doping to demonstrate the diminishing effect of the energy gaps when the periods of the stripe order and the underlying lattice are commensurate of a high rational fraction. Here, $\xi_{c}=4 a$ and $\xi_{s}=8 a$ in order to have a smooth spin wave. Interestingly, the shadow band circling the $\Gamma$ point is very clear.

The condition of half-filled stripes, $x / 2 \epsilon=\frac{1}{2}$, yields an enhanced density of states at Fermi energy. This feature is clearly seen in Figs. 1(b) and 2(b).

\section{B. A disordered stripe phase}

In order to describe superconducting materials, we now consider a quenched random distribution of stripes [16], which should give essentially the correct band structure [17] for slow collective charge dynamics. With $\mathrm{Bi}_{2} \mathrm{Sr}_{2} \mathrm{CaCu}_{2} \mathrm{O}_{8+x}$ in mind, we chose $15 \%$ doping and a mean stripe separation $\ell / a=4$. The ensemble of stripe locations was constructed by taking $x_{n+1}-x_{n}=\ell+\delta$, where the random variable $\delta$ is uniformly distributed between $-3 a$ and $3 a$, which destroys long-range charge and spin order. The spectral density was averaged over five realizations, and we assumed a non-zero temperature, $k_{B} T=t / 10$, which further diminishes finite size effects. We have found that the results do not depend markedly on the choice of ensemble, or the parameters in the effective potential and that the large lattices used in the calculation (linear dimension 184 sites) are essentially selfaveraging. In other words, our results are robust consequences of a disordered stripe array, and are largely independent of other details. (We have not investigated the effects of orientational disorder.)

Figure 3 summarizes shows the $k$-dependence of the spectral density at the Fermi energy, and the quasielectron dispersion along the line $\Gamma-\vec{M}_{1}-X / Y$ for a single, extended domain, with disordered vertical stripes (running in the $\Gamma-\bar{M}_{2}$ direction). Stripe disorder has removed the fine details from the Fermi surface [Fig. 3(a)] which now closely resembles experiments. [2,4]. In particular, near $\bar{M}_{1}$, there is a high density of states and a truly flat "band" at the Fermi energy, extending towards the $\Gamma$ and $X / Y$ points; see Fig. 3(b) and (c). The flatness along the $\Gamma-\bar{M}_{1}$ line is a consequence of both the smearing of the energy gap structure of the ordered system, and the localization of the electronic wave functions in the direction perpendicular to the stripes. The spectral density of the shadow band is reduced so much that it no longer shows up although, on a logarithmic scale, $A_{-}$ looks qualitatively like that of Ref. [3]. The effect of 
vertical stripes at $\bar{M}_{2}$ is completely different: band narrowing in a direction parallel to the stripes leads to an open Fermi surface.

It is clear from the energy dependence of the spectral density that there are no well-defined quasiparticle features near the $\bar{M}_{1}$ point, which is consistent with a widely held view of the normal-state properties of the hightemperature superconductors [18]. This has profound implications for d.c. transport and other low-energy twoparticle properties.

The van Hove singularities of the ordered stripe phase are broadened by disorder. As a result, the density of states $\mathcal{N}\left(\epsilon_{F}\right)$ at the Fermi energy remains enhanced even for $x / 2 \epsilon=\frac{3}{5}$, because it is less sensitive to small variations in doping concentration; see Fig. 3(d).

\section{OPTICAL CONDUCTIVITY}

Finally, consider the real part of the optical conductivity at non-zero energy,

$$
\sigma_{\alpha \alpha}^{\prime}(\omega)=\frac{1-e^{-\hbar \omega / k_{B} T}}{2 \omega} \int_{-\infty}^{\infty} d t e^{i \omega t}\left\langle j_{\alpha}(t) j_{\alpha}(0)\right\rangle
$$

where $j_{\alpha}(t)(\alpha=x, y)$ is the current operator of wave vector $\mathbf{q}=0$ in the Heisenberg presentation [19]. Figure 4 illustrates the optical conductivity for an ordered array of vertical stripes. Transitions from and to the stripeinduced mid-gap states contribute to the pronounced peak at $\hbar \omega \gtrsim t$, while the other transitions yield only a weak feature at $\hbar \omega \sim 3 t$.

Disordering the stripes destroys the momentum conservallion in the perpendicular direction to the stripes. One ronsequence of this that the spectral features in the optical conductivity, shown in Fig. 5, are broadened; there is a rather small weight in the Drude component, so most most of the oscillator strength appears as a broad continuum, extending to zero energy and centered in the neighborhood of $\hbar \omega \sim t$. This property is related to the absence of well-defined quasiparticle features near the $\bar{M}_{1}$ point, as indicated already by the behavior of the spectral density $A_{-}(k, \epsilon)$ near the Fermi energy $\epsilon_{F}$.

\section{DISCUSSION}

In summary, ordered stripe phases have been observed by neutron scattering in non-superconducting $\mathrm{La}_{1.6-x} \mathrm{Nd}_{0.4} \mathrm{Sr}_{x} \mathrm{CuO}_{4}$, and there is strong evidence for disordered and/or fluctuating stripes in $\mathrm{La}_{2-x} \mathrm{Sr}_{x} \mathrm{CuO}_{4-\delta}$. Here we have shown that disordered, or slowly fluctuating stripes in $\mathrm{Bi}_{2} \mathrm{Sr}_{2} \mathrm{CaCu}_{2} \mathrm{O}_{8+x}$ provide a natural explanation for the unusual features of the ARPES data, including the shape of the Fermi surface and the regions of flat bands. It is reasonable to re-examine other experiments on the high-temperature superconductors to see whether they can be better understood in terms of the properties of extended domains with short-ranged stripe order.

Charged stripes are easily pinned by disorder and, at moderate temperatures, they form an essentially quenched-disordered array, which divides the $\mathrm{Cu}-\mathrm{O}$ plane into long thin regions, with weak antiphase coupling between the intervening hole-deficient regions. This provides natural interpretation of NMR experiments [20] which see two distinct species of $\mathrm{Cu}$ nuclei; in our picture, one is in a pinned stripe, the other between the stripes. The fact that the coupling between spin domains is potentially frustrating provides a microscopic justification for the "cluster-spin-glass" phase in samples with $x<15 \%$ [21]. Moreover the creation of dilute metallic stripes can account for the rapid suppression of the Nèel temperature for $x<2 \%$ [22].

\section{ACKNOWLEDGMENTS}

We are grateful to A. Loeser, Z.-X. Shen, J. Tranquada, and B. Wells for stimulating discussions. This work was supported in part by the Division of Materials Science, U. S. Department of Energy under Contracts No. DEAC02-76CH00016 (Brookhaven) and by NSF Grant No. DMR-93-12606 (UCLA). M.I.S was supported in part by the U.S. Department of Energy, Natural Sciences and Engineering Research Council of Canada, and the Ontario Center for Materials Research.

a Present address: Department of Physics and Astronomy, McMaster University, Hamilton, Ontario L8S 4M1, Canada.

- Permanent address: Department of Physics, University of California, Los Angeles, California 90024.

[1] S. A. Kivelson and V. J. Emery, in Strongly Correlated Electronic Materials: The Los Alamos Symposium 1993, edited by K. S. Bedell et al. (Addison-Wesley, Reading, 1994).

[2] D. S. Dessau et al., Phys. Rev. Lett. 71, 2781 (1993).

[3] P. Aebi et al., Phys. Rev. Lett. 72,2757 (1994).

[4] J. Ma et al., Phys. Rev. B51, 9271 (1995).

[5] H. Ding et al. (unpublished).

[6] J. M. Tranquada et al., Nature 375, 561 (1995).

[7] A. R. Moodenbaugh et al., Phys. Rev. B38, 4596 (1988).

[8] Here, wave vectors are expressed in units of $2 \pi$ divided by the lattice spacing.

[9] S.-W. Cheong et al., Phys. Rev. Lett. 67, 1791 (1991); T. E. Mason, G. Aeppli, and H. A. Mook, Phys. Rev. Lett. 68, 1414 (1992); T. R. Thurston et al., Phys. Rev. B46, 9128 (1992). 
[10] Conventionally, the word "domain" is used in two distinct senses. One refers to the region of spins between the charge stripes, the other to larger regions in which one of a discrete set of degenerate ground states is realized. In this paper, we use the name "extended domain" for the second case. Such a domain might be macroscopic or mesoscopic.

[11] J. Zaanen and O. Gunnarsson, Phys. Rev. B40, 7391 (1989); H. Schulz, Phys. Rev. Lett. 64, 1445 (1990); J. A. Verges et al., Phys. Rev. B43, 6099 (1991); M. Inui and P. Littlewood, Phys. Rev. B44, 4415 (1991).

[12] V. J. Emery and S. A. Kivelson, Physica 209C, 597 (1993); U. Löw, V. J. Emery, K. Fabricius, and S. A. Kivelson, Phys. Rev. Lett. 72, 1918 (1994).

[13] L. Chayes, V. J. Emery, S. A Kivelson, Z. Nussinov, and G. Tarjus (in press).

[14] A simple argument using Ginsburg-Landau theory shows that, if the order is driven by the charge, there should be two transitions. with spin ordering occurring at the lower temperature. Recent neutron scattering experiments support this picture. (J. Tranquada, private communication.)

[15] M. Cardona and L. Levy, Photoemission in Solids I (Springer-Verlag. Berlin, 1978).

[16] In principle, a superconducting material could have an ordered array of strupes. However, there is no long-range magnetic order in $\mathrm{La}_{2-x} \mathrm{Sr}_{x} \mathrm{CuO}_{4-\delta}$ and no evidence of charge order in $\mathrm{La}_{2-x} \mathrm{Sr}_{x} \mathrm{CuO}_{4-\delta}$ or $\mathrm{Bi}_{2} \mathrm{Sr}_{2} \mathrm{CaCu}_{2} \mathrm{O}_{8+x}$. Consequently it is appropriate to assume disordered stripes in calculating the band structure. The agreement with experiment indicates that this is a realistic assumption.

[17] Our calculation is similar in spirit to that of A. P. Kampf and J. R. Schrieffer. Phys. Rev. B42, 967 (1990) (KS), although it differs in detail. We compute the effects of static incommensurale spin fluctuations exactly. KS carried out a perturbative ireatment of the dynamics of commensurate spin fiuctuations. The major physical difference is that we focus on states associated with the metallic stripes which reside in the pseudogap found by KS.

[18] For a particulariy insightful exposition of this view, see P. W. Anderson, Science 256, 1526 (1992). See also V. J. Emery and S. A. Kivelson, Phys. Rev. Lett. 74, 3253 (1995); and references therein.

[19] G. D. Mahan, Many-Particle Physics (Plenum Press, New York, 1993).

[20] P. C. Hammel, B. W. Statt, R. L. Martin, S-W. Cheong, F. C. Chou, and D. C. Johnston (unpublished).

[21] J. H. Chou et al., Phys. Rev. B46, 3179 (1992).

[22] F. Borsa et al., Phys. Rev. B52, 7334 (1995); A. CastroNieto and D. Hone (unpublished).
FIG.1 (a) The spectral density $A_{-}$in the first Brillouin zone integrated over an energy window $\Delta \epsilon=t / 30$ about $\epsilon F$ and (b) the density of states $\mathcal{N}(\epsilon)$ as a function of energy $\epsilon$ for an ordered array of vertical stripes of period $\ell=4 a$ with $\frac{1}{8}$ doping. The size of a circle, denoting a Fermi-surface crossing at a given value of $k$, shows the relative magnitude of $A\left(\mathbf{k}, \epsilon_{F}\right)$. The parameters specifying the effective potential are $\rho_{0}=-t / 2, S_{0}=2 t, \xi_{c}=a$, and $\xi_{s}=2 a$.

FIG.2 (a) The spectral density $A_{-}$in the first Brillouin zone integrated over an energy window $\Delta \epsilon=t / 30$ about $\epsilon_{F}$ and (b) the density of states $\mathcal{N}(\epsilon)$ as a function of energy $\epsilon$ for an ordered array of vertical stripes of period $\ell=10 a$ with $\frac{1}{20}$ doping. The size of a circle, denoting a Fermi-surface crossing at a given $\mathbf{k}$, shows the relative magnitude of $A\left(k, \epsilon_{F}\right)$. The parameters specifying the effective potential are $\rho_{0}=-t / 2, S_{0}=2 t, \xi_{c}=4 a$, and $\xi_{s}=8 a$.

FIG.3 (a) The spectral density $A_{-}$in the first Brillouin zone integrated over an energy window $\Delta t=t / 30$ about $\epsilon_{F}$, (b) the dispersion relation, (c) the corresponding spectral density of the highest-energy occupied "band" as a function of $k$ along the $\Gamma-\bar{M}_{1}-X / Y$ line, and (d) the density of states $\mathcal{N}(\epsilon)$ as a function of energy $\epsilon$. The band is determined by broadening the energy $\delta$-functions by a Lorentzian of the full width of $t / 4$ at half maximum and finding the highest-energy maximum of $A_{-}$. The results are for a disordered array of vertical stripes with the mean separation of $\ell=4 a$ with $15 \%$ doping at the temperature $k_{B} T=t / 10$. The rest of the parameters are $\rho_{0}=-t / 2, S_{0}=2 t, \xi_{c}=a$, and $\xi_{s}=2 a$.

FIG.4 The real (absorptive) part of the optical conductivity $\sigma_{\|, \perp}^{\prime}(\omega)$ perpendicular (solid line) and parallel (dashed line) to an ordered array of vertical stripes with the separation of $\ell=4 a$ at $\frac{1}{8}$ doping at zero temperature. The stripe configuration and the parameters are the same as in Fig. 1.

FIG.5 The real (absorptive) part of the optical conductivity $\sigma_{\|, \perp}^{\prime}(\omega)$ perpendicular (solid line) and parallel (dashed line) to a disordered array of vertical stripes with the mean separation of $\ell=4 a$ at $15 \%$ doping at zero temperature. The stripe ensemble and the parameters are the same as in Fig. 3. 

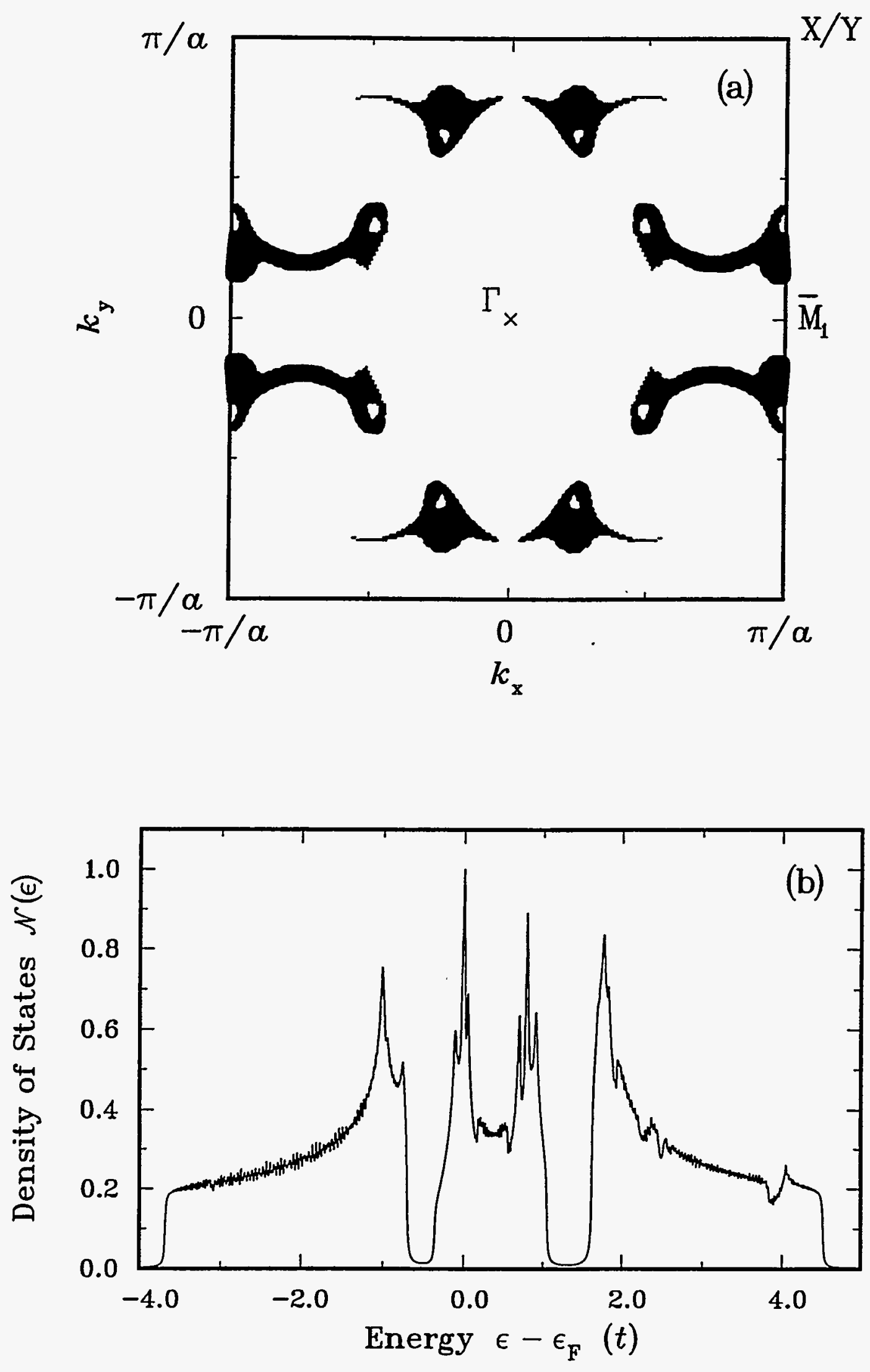

Fig. 1 

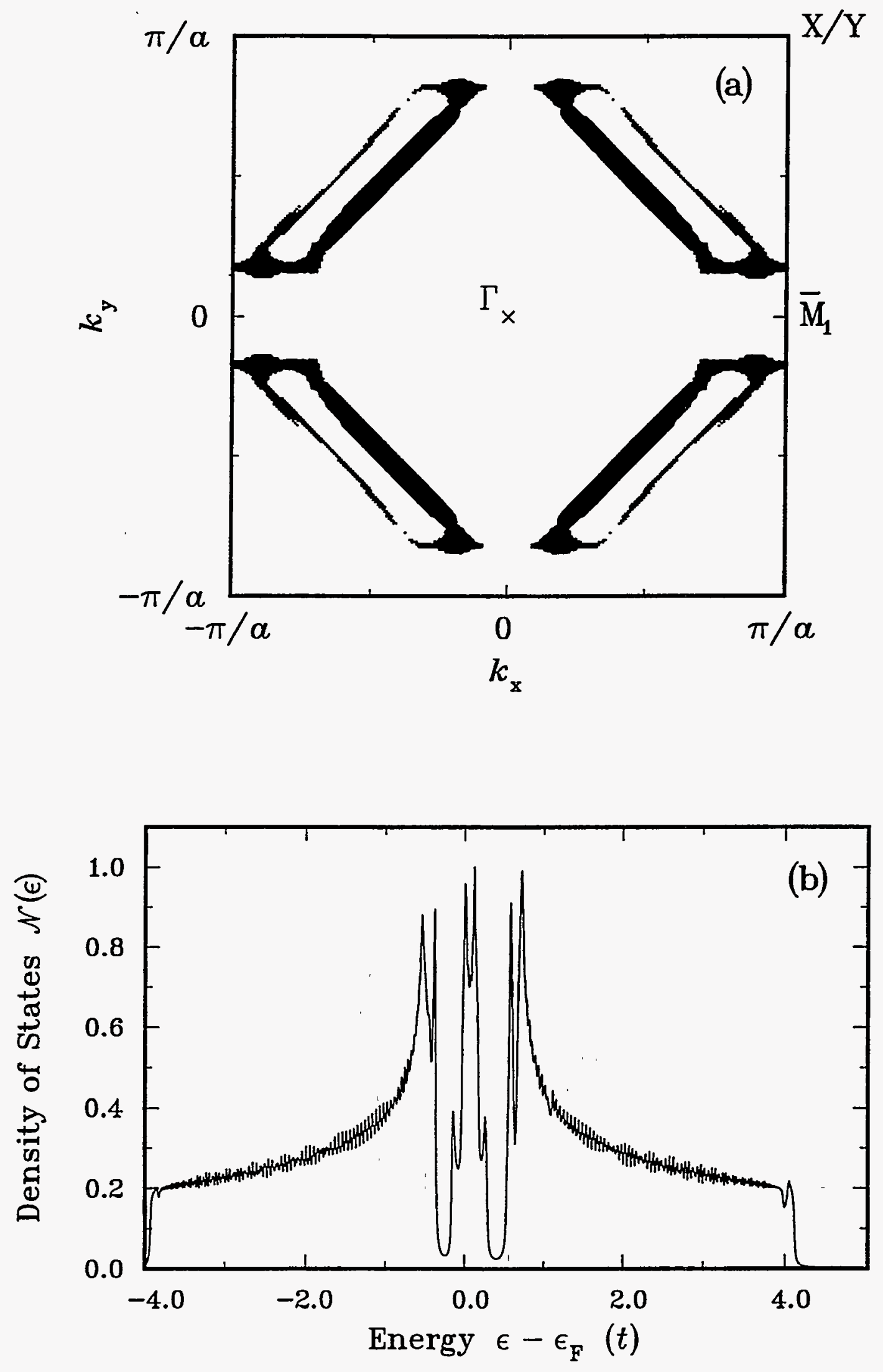

Fig. 2 

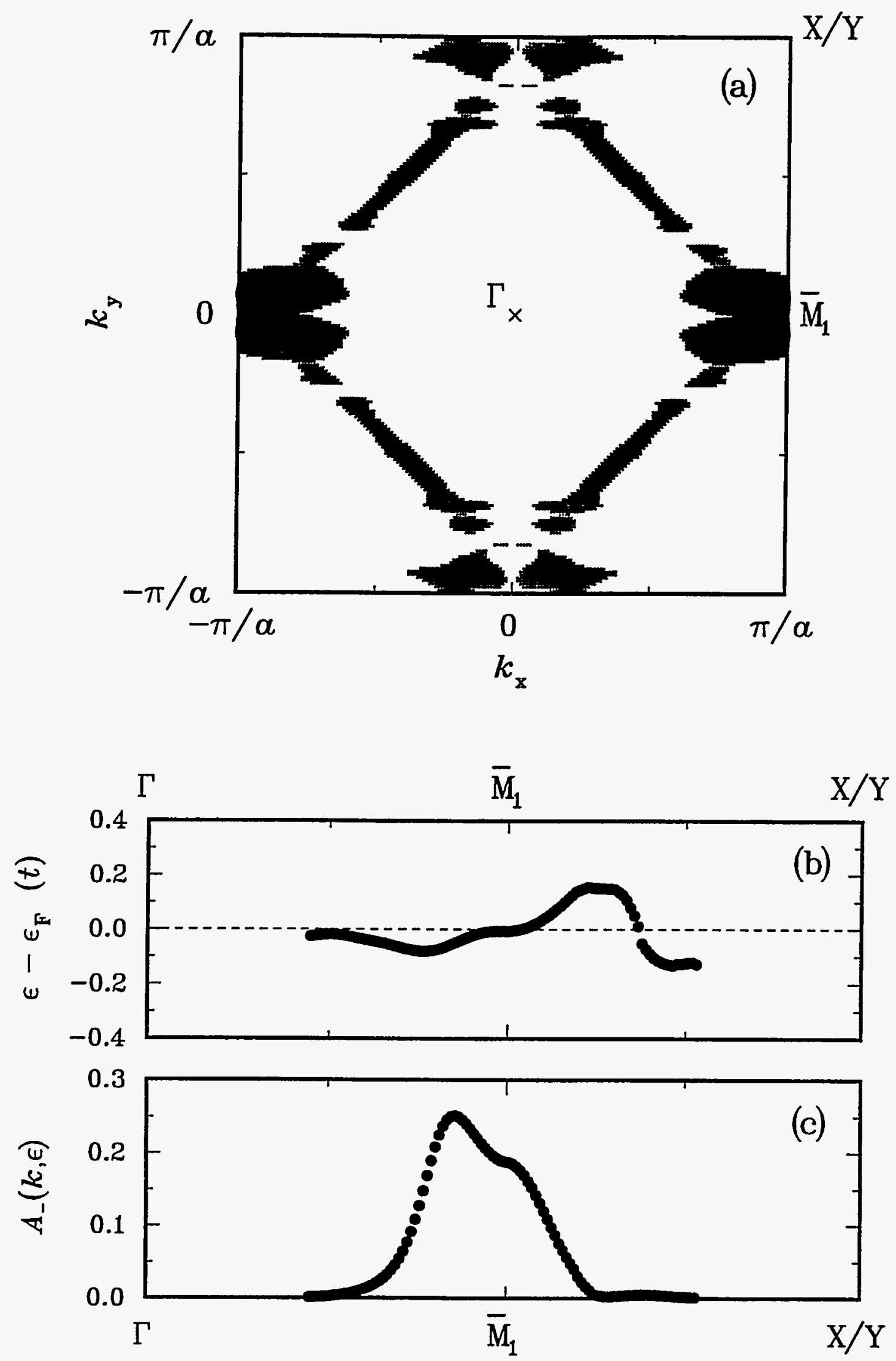

Wave vector $k$

Fig. 3 


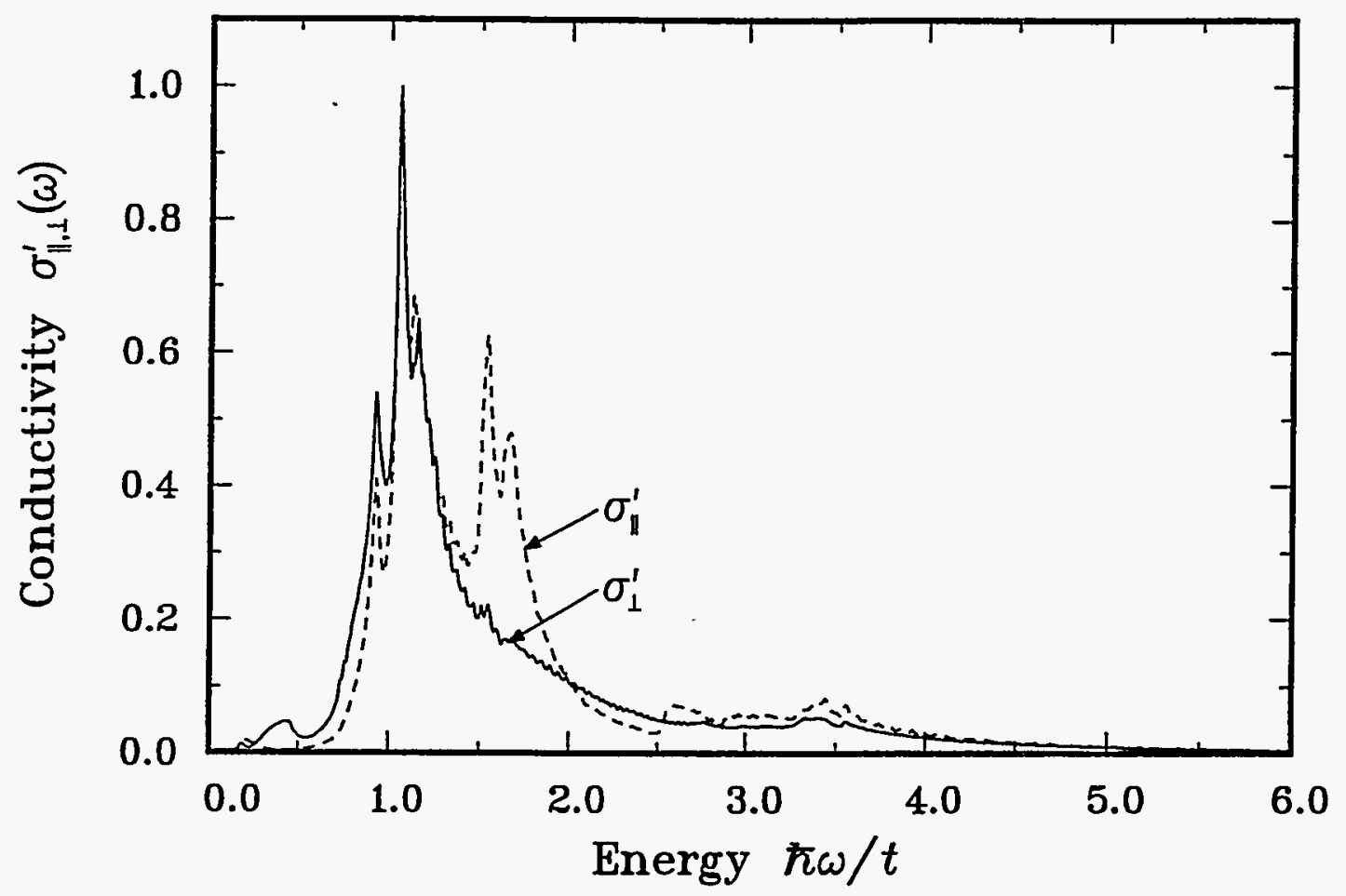

Fig. 4 


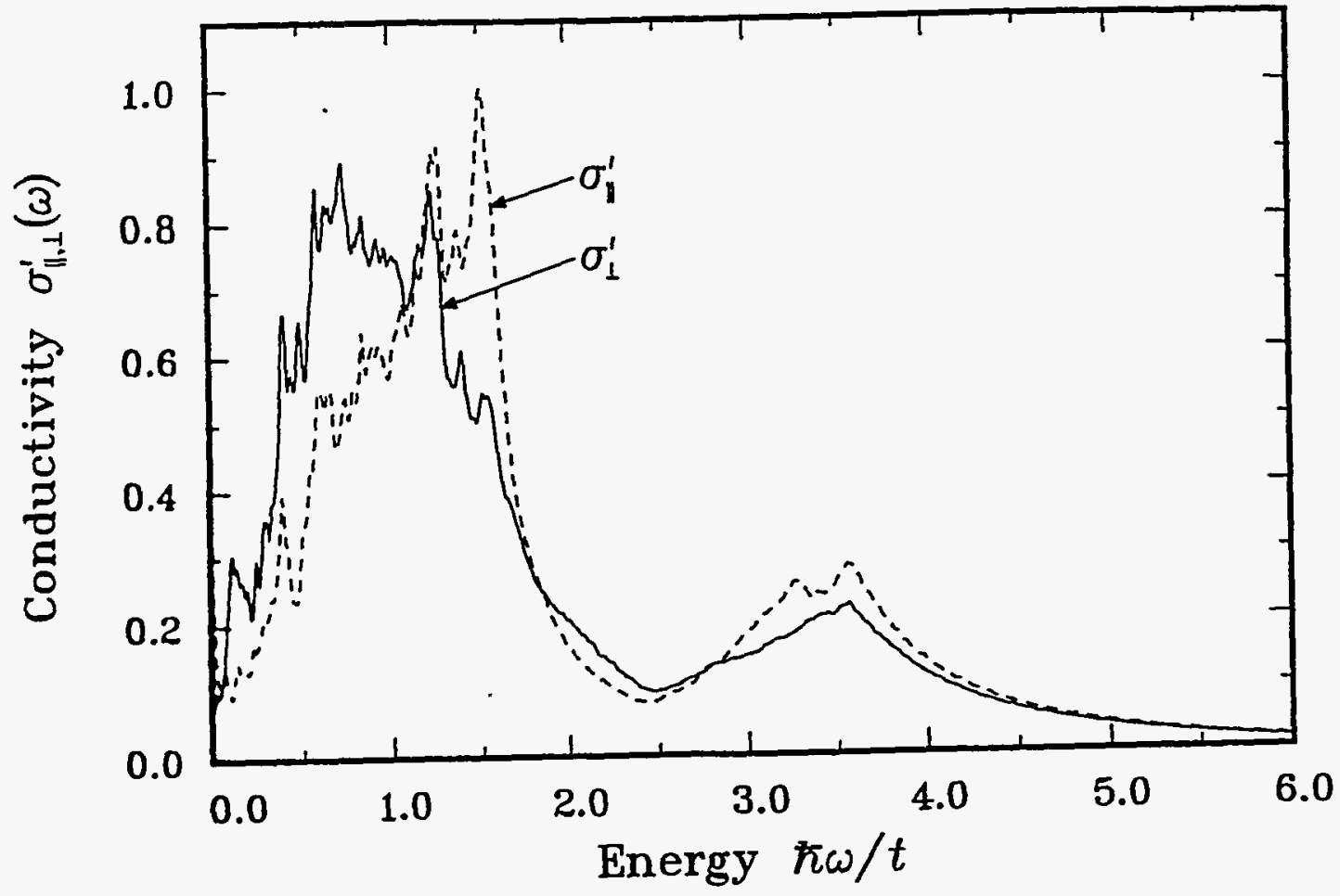

Fig. 5

\section{DISCLAIMER}

This report was prepared as an account of work sponsored by an agency of the United States Government. Neither the United States Government nor any agency thereof, nor any of their employees, makes any warranty, express or implied, or assumes any legal liability or responsibility for the accuracy, completeness, or usefulness of any information, apparatus, product, or process disclosed, or represents that its use would not infringe privately owned rights. Reference herein to any specific commercial product, process, or service by trade name, trademark, manufacturer, or otherwise does not necessarily constitute or imply its endorsement, recommendation, or favoring by the United States Government or any agency thereof. The views and opinions of authors expressed herein do not necessarily state or reffect those of the United States Government or any agency thereof. 


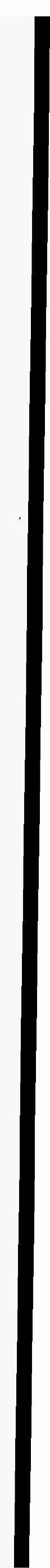

